\title{
Microalgal Culture for Chlorella sp. using a Hollow Fiber Membrane Module
}

\author{
Yoshihiko Sano ${ }^{1 *}$, Akihiko Horibe ${ }^{2}$, Naoto Haruki ${ }^{2}$ and Yugo Okino ${ }^{2}$
}

${ }^{1}$ Department of Mechanical Engineering, Shizuoka University, 3-5-1 Johoku, Naka-ku, Hamamatsu, 432-8561, Japan

${ }^{2}$ Graduate School of Natural Science and Technology, Okayama University, 3-1-1 Tsushima-naka, Kita-ku, Okayama, 700-8530 Japan

\begin{abstract}
A hollow fiber culture system has been proposed for supplying the carbon dioxide to the microalgae, to replace the conventional air bubbling system which has been adopted to supply carbon dioxide in most conventional microalgal culture. In order to examine the usefulness of hollow fiber membranes for the microalgal culture, the microalgal growth rate for Chlorella sp. and the effective mass transfer coefficient of carbon dioxide through the hollow fiber membranes have been measured using the proposed photobioreactor filled with hollow fibers. The microalgal growth rate using hollow fiber membranes was found to be three times greater than that observed in the conventional non-membrane photobioreactor. An experimental investigation has been conducted so as to evaluate the effect of the volume flow rate of the carbon dioxide and its concentration of the feed air through the hollow fibers on the microalgal growth rate. The present study clearly indicates that the hollow fiber membrane is quite useful for the microalgae culture in terms of enhancing both microalgal growth rate and dissolution rate of the carbon dioxide.
\end{abstract}

Keywords: Microalgal culture; Membrane transport; Hollow fiber membrane; Microalgal growth rate

\section{Nomenclature}

$a_{i}$ : Specific surface area $[1 / \mathrm{m}]$

$A_{\text {int }}:$ Interface area between the liquid and membrane phases $\left[\mathrm{m}^{2}\right]$

$c$ : Total carbon concentration $\left[\mathrm{kg} / \mathrm{m}^{3}\right]$

$c_{\text {feed }}: \mathrm{CO}_{2}$ concentration of mixed air [\%]

$d_{m}$ : Outer diameter of the hollow fiber [m]

$D$ : Inner diameter of the hollow fiber membrane device $[\mathrm{m}]$

$D_{l}$ : Carbon dioxide diffusion coefficient $\left[\mathrm{m}^{2} / \mathrm{s}\right]$

$h_{g}$ : Interstitial mass transfer coefficient for the gas-membrane interface $[\mathrm{m} / \mathrm{s}]$

$h_{l}$ : Interstitial mass transfer coefficient for the membrane-liquid interface $[\mathrm{m} / \mathrm{s}]$

$h_{e f f}:$ Effective mass transfer coefficient $[\mathrm{m} / \mathrm{s}]$

$L_{x}:$ Light intensity $[\mathrm{lx}]$

M: Microalgal concentration $\left[\mathrm{kg} / \mathrm{m}^{3}\right]$ side $[-]$

$n_{i}$ : Unit vector pointing outward from the liquid side to membrane

$Q$ : Volume flow rate of the feed gas $\left[\mathrm{m}^{3} / \mathrm{s}\right]$

$t$ : Time $[\mathrm{s}]$

T: Temperature $\left[{ }^{\circ} \mathrm{C}\right]$

$t_{m}:$ Membrane thickness $[\mathrm{m}]$

$V$ : Representative elementary volume $\left[\mathrm{m}^{3}\right]$

$V_{i}$ : Total volume of liquid phase $\left[\mathrm{m}^{3}\right]$

\section{Introduction}

Several attempts have been successfully made to produce the biomass fuel from the microalgae [1,2]. Some microalgae are capable of producing the biofuel which is carbon neutral to the environment, absorbing light, nutrition and carbon dioxide. Microalgae have a high oil production capacity as compared with common food sources, such as corn and soybean. There is no competition with food industry since microalgae are not a common food source. A report published by Chisti [1] indicates that the production efficiency of microalgae is about ten times as high as palm oil. Moreover, microalgae do not require extensive arable fields to cultivate them and they can be cultivated irrespectively of the season.

Various microalgal reactors have been developed in order to cultivate microalgae [2]. Especially, open ponds are the most widely applied in industrial processes [3], which are composed of long single or multiple loop channels placed outdoors. Open ponds are possible to produce microalgae at low cost as compared with other systems by taking account of building and maintenance costs. On the other hand, closed photobioreactors have drawn attention in views of controlling temperature and light intensity at favorable conditions for microalgal species. Moreover, photobioreactors allow us to maintain a high biomass production and reduce the risk of contamination [4]. Various photobioreactors illuminated naturally or artificially lit were proposed such as inside open ponds [5,6], flat-plate [7], airlift [8,9] and tubular [10].

A number of researchers investigated the effect of important factors on microalgal culture, such as light, minerals, temperature and

*Corresponding author: Yoshihiko Sano, Department of Mechanical Engineering, Shizuoka University, 3-5-1 Johoku, Naka-ku, Hamamatsu, 432-8561, Japan, Tel: +81-534-478-1048; Fax: +81-534-478-1048; E-mail: sano.yoshihiko@shizuoka-u.ac.jp

Received February 22 2016; Accepted March 07, 2016; Published March 09, 2016

Citation: Sano Y, Horibe A, Haruki N, Okino Y (2016) Microalgal Culture for Chlorella sp. using a Hollow Fiber Membrane Module. J Membra Sci Technol 6: 147. doi:10.4172/2155-9589.1000147

Copyright: ( 2016 Sano Y, et al. This is an open-access article distributed under the terms of the Creative Commons Attribution License, which permits unrestricted use, distribution, and reproduction in any medium, provided the original author and source are credited. 
$\mathrm{CO}_{2}$ concentration. Several papers [11-13] indicated that light intensity is one of the most important limiting factors since microalgae carry out photosynthesis. Thus, microalgae culture systems are illuminated by artificial light or solar light. On the other hand, the optimal temperature for microalgae cultures is generally between 20 and $25^{\circ} \mathrm{C}$, although this may vary with the composition of the culture medium and the microalgal species [14]. Moreover, it is also well known that the carbon dioxide also plays an important role in microalgal culture through investigations for the effect of $\mathrm{CO}_{2}$ concentration on the microalgal growth rate in photobioreactors [15-17]. Some microalgal production facilities use combustion gases of fossil fuels so as to increase productivity of the microalgae. Chiu et al. [16] introduced the mixed air controlled at high $\mathrm{CO}_{2}$ concentration $10-15 \% \mathrm{v} / \mathrm{v}$ into microalgal culture of Chlorella sp. Furthermore, Hirata et al. [17] revealed that Chlorella sp. can grow in up to $40 \% \mathrm{CO}_{2}$ concentration at $30^{\circ} \mathrm{C}$.

In most conventional microalgae culture, an air bubbling has been adopted to supply the carbon dioxide. However, in the medical field, especially in most blood oxygenators, hollow fiber membranes are widely used to supply gases into the blood. According to Carvalho et al. [18], the membrane makes it possible to diffuse the carbon dioxide into the microalgae culture in lower gas pressures, as no need to counterbalance hydrostatic heads. Photobioreactors using membranes have been reported for culture of mammalian cells [19], Chlamydomonas reinhardtii [20], P. tricornutum in seawater [21] and Anabaena Variabilis [22].

In this study, a hollow fiber culture system has been proposed for supplying the carbon dioxide to the microalgae for Chlorella sp. In order to examine the usefulness of hollow fiber membranes for the microalgal culture, we shall measure the microalgal growth rate for chlorella sp. and the effective mass transfer coefficient of the carbon dioxide through the hollow fiber Silicon tubes exploiting the proposed photobioreactor. Moreover, the microalgal growth rate using hollow fiber membranes are compared against that of a non-membrane photobioreactor. Subsequently, we shall evaluate the effect of the volume flow rate and $\mathrm{CO}_{2}$ concentration of the feed air through the hollow fibers on the microalgal growth rate.

\section{Materials}

\section{Culture of Chlorella sp.}

The green alga Chlorella sp., in which the diameter is about 2-10 $\mu \mathrm{m}$ spherical, has been employed in this study. Chlorella sp. has been focused on a potential source of supplements and energy source because they have high photosynthetic efficiency. It is well known that Chlorella $s p$. can grow in high $\mathrm{CO}_{2}$ concentration in excess of $10 \%$ [16,17]. In this study, the culture medium was adopted Hyponex medium widely used for the plant growth so as to take account of industrial applications of microalgal production.

Previously, Chlorella sp. was cultivated in $100 \mathrm{~mL}$ glass beakers illuminated by the white light emitting diode (LED), under the several temperature $20-30^{\circ} \mathrm{C}$, the dilution rate of Hyponex medium, the light intensity 600 6000lx at the top of microalgal culture in beakers. It was found that the microalgal growth rate of Chlorella sp. is the highest under the condition at temperature $26^{\circ} \mathrm{C}$ and the light intensity $2000 \mathrm{~lx}$, conducting a series of preliminary experiments in beakers. Moreover, the culture medium concentrations were composed (in mgL-1): $\mathrm{TN}=24$, $\mathrm{NH}_{4}=11.6, \mathrm{NO}_{3}=4.2, \mathrm{P}_{2} \mathrm{O}_{5}=40, \mathrm{~K}_{2} \mathrm{O}=20, \mathrm{MgO}=0.02, \mathrm{MnO}=0.004$, $\mathrm{B}_{2} \mathrm{O}_{3}=0.02$. Thus, these conditions were applied to cultivate Chlorella $s p$. in the photobioreactor using the hollow fiber membranes in this study. The microalgal concentration was determined through its correlation to absorbance of the dry weight for chlorella $s p$. at $690 \mathrm{~nm}$ by using an absorption spectrometer (AS ONE, ASV11D).

\section{Hollow fiber devices}

Figure 1 schematically shows a hollow fiber membrane device for the microalgal culture, in which thousands of small silicon tubes are arranged so as to allow to the carbon dioxide diffuse into the microalgal culture through the inside of the hollow fibers. Two types of hollow fiber devices, M60-3000, M40-3000 produced by Nagayanagi Kogyo (Japan), were applied for the photobioreactor of chlorella sp. Geometric characteristics of the membrane module are provided by the manufacturer in Table 1. All of hollow fiber membranes are made of Silicon, the thickness of membranes varies 40-60 $\mu \mathrm{m}$ in M40-3000 and M60-3000. Silicon membranes employed in this study can be classified into a non-porous membranes. The diffusion process of $\mathrm{CO}_{2}$ through this silicon membrane is that the $\mathrm{CO}_{2}$ molecules are adsorbed on the membrane interface, and then diffuse through the membrane, finally dissolved to the liquid side. Permeability is closely related to the partial pressure. The pressure inside of the hollow fiber can be considered as the atmospheric pressure since the outlet of the hollow fiber membrane module is opened in this study. Therefore, the partial pressure would depend on $\mathrm{CO}_{2}$ concentration.

\section{Methods}

\section{Microalgae culture using a hollow fiber membrane device}

The experimental set-up used for the hollow fiber culture system is schematically illustrated in Figure 2. The hollow fiber membrane device was placed in the tunnel illuminated by eight LED lights. The light intensity was adjusted to 2000lx at the surface of the cylinder for photobioreactor using a hollow fiber membrane device. The temperature of the mixed air supplied by an air pump was adjusted to $26^{\circ} \mathrm{C}$ as it passes through a heat exchanger in the isothermal bath. $\mathrm{CO}$ concentration of the mixed air was controlled by varying flow rate of air and $\mathrm{CO}_{2}$ gas from $\mathrm{CO}_{2}$ tank. The mixed air, which is controlled to the arbitrary temperature and concentration, is fed into the hollow fibers for photobioreactor, and then the carbon dioxide penetrates through the hollow fiber membrane and diffuses into microalgal culture filled
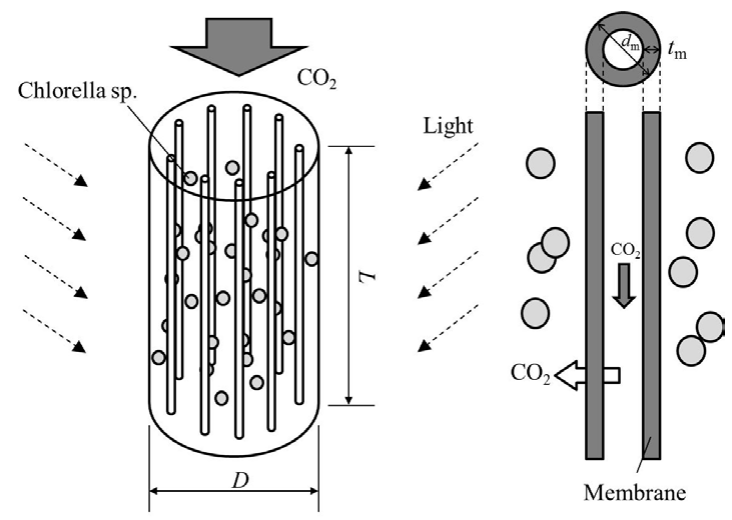

Figure 1: Photobioreactor using a hollow fiber membrane device.

\begin{tabular}{|l|c|c|c|c|c|}
\hline & $\boldsymbol{N}[-]$ & $\boldsymbol{d}_{\boldsymbol{m}}[\mathrm{mm}]$ & $\boldsymbol{t}_{\boldsymbol{m}}[\boldsymbol{\mu m}]$ & $\boldsymbol{D}[\mathbf{m m}]$ & $\boldsymbol{L}[\mathbf{m m}]$ \\
\hline M40-3000 & 3000 & 0.25 & 0.04 & 31 & 135 \\
\hline M60-3000 & 3000 & 0.32 & 0.06 & 31 & 135 \\
\hline
\end{tabular}

Table 1: Geometric characteristics of hollow fiber membrane devices. 


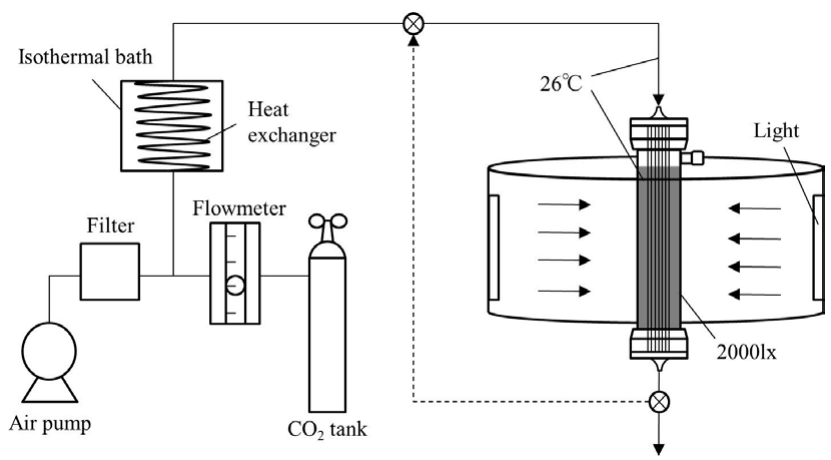

Figure 2: Hollow fiber culture system.

outside of hollow fiber membranes. An air from one end of a hollow fiber membrane device can be fed back to this device the using a threeway valve located posteriorly in a hollow fiber membrane device. However, in this fundamental study, we focused on microalgal culture when an exhausted air was not fed back to a hollow fiber membrane device again.

In order to examine the usefulness of the proposed photobioreactor filled with hollow fiber membranes, we shall conduct an experiment on microalgal culture to measure the microalgal concentration of chlorella $s p$. Then, the microalgal growth rate of the proposed photobioreactor using hollow fiber membranes are compared against that of a nonmembrane photobioreactor, which is a cylindrical acrylic tank as large as hollow fiber membrane devices. Subsequently, the effects of the volume flow rate and $\mathrm{CO}_{2}$ concentration of the feed mixed air through the hollow fibers on the microalgal culture were investigated to verify the usefulness of the microalgal growth rate in the proposed photobioreactor.

\section{Diffusion of $\mathrm{CO}_{2}$ through hollow fiber membranes}

The overall mass transfer coefficient of the carbon dioxide from the hollow fibers to the pure water filled outside the hollow fibers was estimated by exploiting the hollow fiber culture system illustrated in Figure 2. Pure water was filled in the liquid phase of a hollow fiber membrane device (namely shell side of the membrane), and then pure $\mathrm{CO}_{2}$ gas is fed into the inner side of hollow fibers. Temperature of liquid and gas phases is controlled $26^{\circ} \mathrm{C}$ the same condition as for microalgal culture in this study. Total carbon concentration was measured using a multi-water quality meter (MM60, ToaDKK) with a carbonic acid electrode (CE-2041, ToaDKK).

The volumetric mass transfer rate of the carbon dioxide through the membranes may be given in terms of the effective mass transfer coefficient $h_{\text {eff }}$ as follows:

$$
\frac{1}{V} \int_{A_{l i t t}} D_{l} \frac{\partial c}{\partial x_{j}} n_{l j} d A=a_{l} h_{e f f}\left(\bar{c}^{*}-\bar{c}\right)
$$

where $\mathrm{n}_{l j}$ is the unit vector pointing outward from the liquid side to membrane side, while $A_{l_{i u t}}$ and $a_{l}$ are the interfacial area and the specific surface area between the liquid and membrane phases. $D_{l}$ is the carbon dioxide diffusion coefficient in the liquid phase. The bulk average of a certain local variable $\varphi$ in the liquid phase is defined as

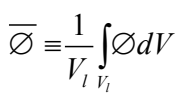

such that $\bar{c}$ is the intrinsic average of the total $\mathrm{CO}_{2}$ concentration in the liquid phase, while $\bar{c} *$ is its equilibrium concentration, namely, the intrinsic average of the total $\mathrm{CO}_{2}$ concentration in the liquid phase when the liquid phase is in equilibrium with the gas phase. $V_{l}$ is the volume space which the liquid phase occupies, thus the porosity $\varepsilon_{l} \equiv$ $V_{l} / V$ is the volume fraction of the liquid space.

Further simplification is possible as one notes the difference in the mass transfer resistances among the gas phases, membrane phases, liquid phases and the surface of the cells for the microalgal culture. According to Goldman et al. [23] and Becker [24], the resistance at the gas-liquid interface is much larger than at the boundary layer of the cells. Thus, the total mass transfer resistance between the liquid and gas phases is defined as,

$$
\frac{1}{h_{e f f}}=\left(\frac{d_{m}}{d_{m}-t_{m}}\right) \frac{1}{h_{g}}+\frac{d_{m}}{D_{m}} \ln \left(\frac{d_{m}}{d_{m}-t_{m}}\right)+\frac{1}{h_{l}}
$$

where $h_{g}$ and $h_{l}$ are the interstitial mass transfer coefficient for the gas-membrane interface and the membrane-liquid interface, $D_{m}$ is the carbon dioxide diffusion coefficient in the membrane phase. We shall consider an unsteady mass balance equation for the carbon dioxide passing through membrane as follows:

$$
V_{l} \frac{d \bar{c}}{d t}=A_{l} h_{e f f}(\bar{c} *-\bar{c})
$$

which, with the initial condition, namely, $\bar{c}=\left.\bar{c}\right|_{t=0}$ already measured, gives us an exponential time dependent of the total $\mathrm{CO}_{2}$ concentration for the liquid phase:

$$
\frac{\bar{c}-\left.\bar{c}\right|_{t=0}}{\bar{c} *-\left.\bar{c}\right|_{t=0}}=1-\exp \left(-\frac{A_{l} h_{e f f}}{V_{1}} t\right)=1-\exp \left(-\frac{a_{l} h_{e f f}}{\varepsilon_{l}} t\right)
$$

The effective mass transfer coefficient is adjusted by fitting the measured temporal development of the total $\mathrm{CO}_{2}$ concentration in the liquid phase to the foregoing equation.

\section{Results}

\section{Diffusion of $\mathrm{CO}_{2}$ through hollow fiber membranes}

All measured values of the total $\mathrm{CO}_{2}$ concentration in the liquid phase using the hollow fiber membrane devices are plotted against the elapse time in Figure 3 for the case of $T=26^{\circ} \mathrm{C}, V_{l}=50 \mathrm{ml}$, using pure water and pure $\mathrm{CO}_{2}$ gas. As shown in this figure, the total $\mathrm{CO}_{2}$ concentration in the liquid phase suddenly rises after the feed of $\mathrm{CO}_{2}$ gas to the hollow fibers, and then achieves the critical value for the equal amount of pure $\mathrm{CO}_{2}$ gas. The volumetric effective mass transfer coefficient $a_{l} h_{e f f} / \varepsilon_{l}$ and the effective mass transfer coefficient $h_{\text {eff }}$ are

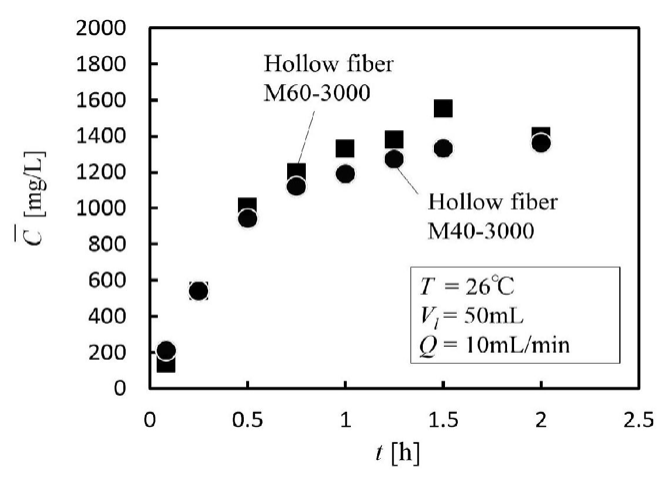

Figure 3: Development of total $\mathrm{CO}_{2}$ concentration. 
tabulated in Table 2, estimated by fitting the measured total $\mathrm{CO}_{2}$ concentration developments to the $\mathrm{CO}_{2}$ concentration profile obtained from an equation 5 . In the same table, the experimental data reported by Carvolho et al. [18] are tabulated for reference. As can be seen from Table 2, the values of the volumetric effective mass transfer coefficient in present devices are higher than those of HIFO and HIFI provided by Carvolho et al. [18] since specific surface area of present devices is larger than that of HIFO and HIFI. Therefore, present hollow fiber devices can easily be adapted to control the $\mathrm{CO}_{2}$ concentration in microalgal culture with varying the pressure of inner side of membrane or the number of hollow fibers.

Effect of the volume flow rate of the feed gas on the carbon diffusion is illustrated in Figure 4. The difference in the two sets of plots is indiscernible, which indicates that the volume flow rate of the feed gas is sufficiently larger than carbon diffusion rate through hollow fiber membranes in this study. Therefore, in order to efficiently supply $\mathrm{CO}_{2}$ to microalgae culture, a feedback system of an excess gas may be adopted in an industrial application.

\section{Microalgae culture using a hollow fiber membrane device}

The picture of the cultured microalgae in the hollow fiber membrane device (M40-3000) after 120 hours is shown in Figure 5, in which Chlorella sp. is cultured in the space formed by the hollow fibers. In Figure 6, the development of the cell concentrations $M$ using hollow fiber membrane device M40-3000 are compared against that using a non-membrane photobioreactor in which mixed air is fed by air bubbling at the bottom. $\mathrm{CO}_{2}$ concentration of mixed air was set to 20 $\% \mathrm{v} / \mathrm{v}$. Experimental period was set to 5 days in which cell concentrations may increase linearly, although it is known that the microbial growth can be expressed an exponential function. As can be seen in Figure 6, the microalgal growth rate using hollow fiber membrane device is found to be three times greater than that of a non-membrane photobioreactor. This high cultivation rate of microalgae using hollow fiber membranes would be due to reduction of shear stress associated with supplying

\begin{tabular}{|l|l|l|l|l|l|}
\hline Device & $\boldsymbol{a}_{\boldsymbol{l}} \boldsymbol{h}_{\text {eff }} / \boldsymbol{\varepsilon}_{\boldsymbol{l}}\left[\mathbf{m i n}^{-1}\right]$ & $\boldsymbol{A}\left[\mathbf{m}^{2}\right]$ & $\boldsymbol{a}_{\boldsymbol{l}}\left[\mathbf{m}^{-1}\right]$ & $\boldsymbol{h}_{\text {eff }}[\mathbf{m} / \mathbf{s}]$ & Reference \\
\hline M40-3000 & $3.17 \times 10^{-2}$ & 0.20 & 3160 & $1.34 \times 10^{-7}$ & Present study \\
\hline M60-3000 & $3.41 \times 10^{-2}$ & 0.30 & 4050 & $9.50 \times 10^{-2}$ & Present study \\
\hline HIFO & $1.48 \times 10^{-2}$ & 1.70 & 1700 & $1.45 \times 10^{-2}$ & Carvolho et al. [18] \\
\hline HIFI & $1.33 \times 10^{-2}$ & 0.14 & 140 & $1.59 \times 10^{-2}$ & Carvolho et al. [18] \\
\hline
\end{tabular}

Table 2: Effective mass transfer coefficient of the carbon dioxide.

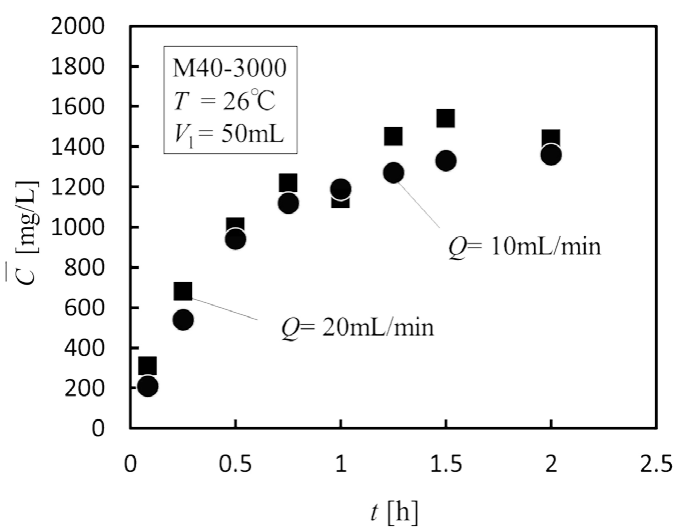

Figure 4: Effect of the volume flow rate of the feed gas on the carbon diffusion through hollow fiber membranes.

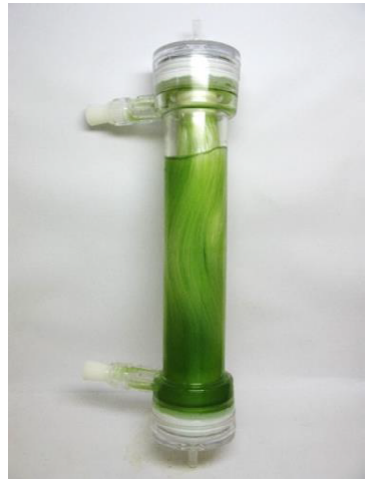

Figure 5: Picture of the cultured microalgae in the hollow fiber membrane device (M40-3000) $\left(T=26^{\circ} \mathrm{C}, V l=50 \mathrm{~mL}, L x=2000 \mathrm{~lx}, Q=25 \mathrm{~mL} / \mathrm{min}\right.$, after $\left.t=120 \mathrm{~h}\right)$.

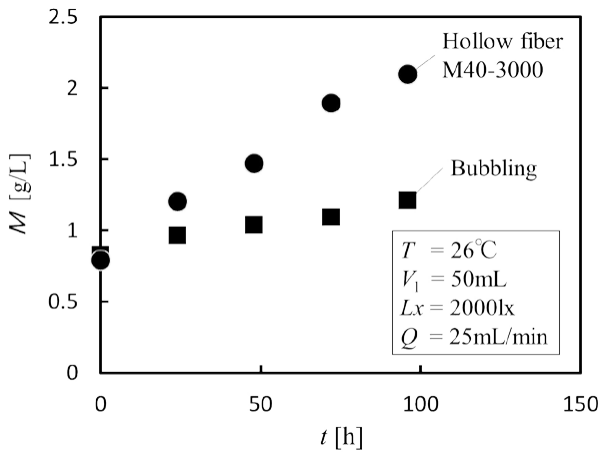

Figure 6: Development of cell concentration for comparison between the hollow fiber device and air bubbling.

gas, since carbon dioxide concentration in the culture medium reached in equilibrium regardless of both supply method after two hours. If adopting the feedback of mixed air, it is possible to significantly reduce the amount of carbon dioxide. These data indicate the usefulness of the hollow fiber module in microalgal culture in terms of cultivation rate and amount of carbon dioxide.

The values of the cell concentrations obtained from M40-3000 and M60-3000 are plotted against the elapse time in Figure 7. The microalgal growth rate of M40-3000 is larger than that of M60-3000, despite that the difference in dissolution behavior of carbon dioxide were indiscernible. Arrangement of hollow fibers appears to be important factors for microalgal culture in terms of the light attenuation through the bundle of hollow fibers. Actually, the microbial growth was not observed in the module with 6000 hollow fibers in preliminary experiments.

Effect of the volume flow rate of the feed mixed gas on the microalgal growth rate is illustrated in Figure 8. It is found that the variation of the flow rate does not affect the microalgal growth rate. Moreover, the effect of $\mathrm{CO}_{2}$ concentration of mixed air on the microalgal growth rate is illustrated in Figure 9. There exists an optimum carbon dioxide concentration for each microalgae, and then the appropriate conditions will lead to the further cultivation rate for the microalgal culture using the hollow fiber membrane device. Thus, the hollow fiber membrane is usefulness for the microalgae culture in terms of the microalgal growth rate and the dissolution of the carbon dioxide. 
Citation: Sano Y, Horibe A, Haruki N, Okino Y (2016) Microalgal Culture for Chlorella sp. using a Hollow Fiber Membrane Module. J Membra Sci Technol 6: 147. doi:10.4172/2155-9589.1000147

Page 5 of 6

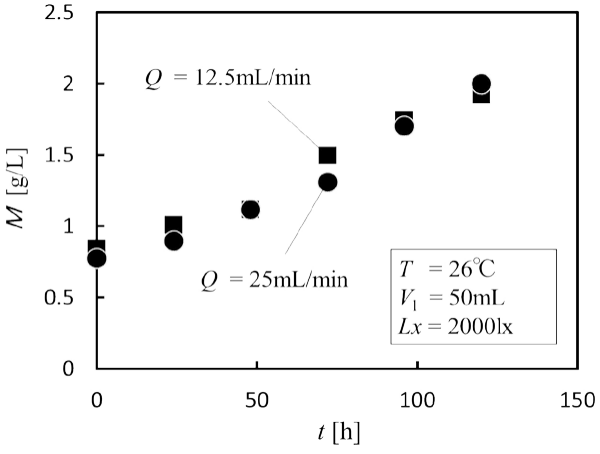

Figure 7: Microalgal growth rate obtained from M40-3000 and M60-3000

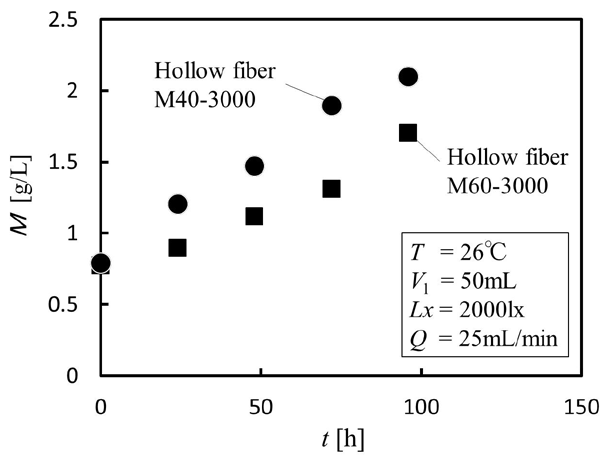

Figure 8: Effect of the volume flow rate of the feed mixed air on the microalgal growth rate.

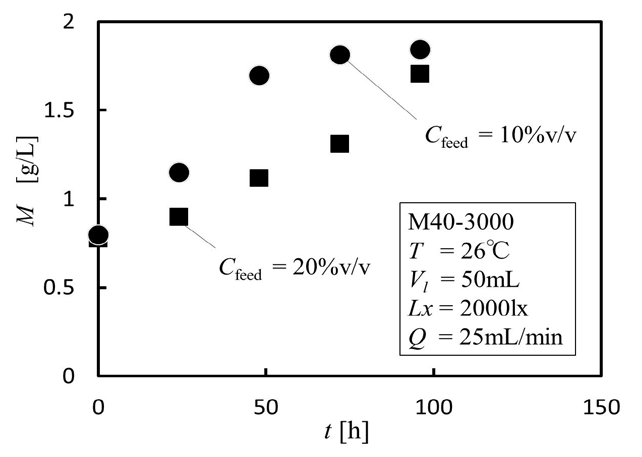

Figure 9: Effect of $\mathrm{CO}_{2}$ concentration of mixed air on the microalgal growth rate.

\section{Conclusions}

The hollow fiber culture system was proposed for supplying the carbon dioxide to the microalgae for Chlorella sp. The overall mass transfer coefficient of the carbon dioxide between pure $\mathrm{CO}_{2}$ gas inside the hollow fibers and pure water filled outside the hollow fibers was estimated by exploiting the hollow fiber culture system. The microalgal growth rate using hollow fiber membranes is found to be three times greater than that of a non-membrane photobioreactor. Moreover, the appropriate conditions of $\mathrm{CO}_{2}$ concentration and the number of hollow fibers will lead to the further growth rate for the microalgal culture using the hollow fiber membrane device. The present study clearly indicates that the hollow fiber membrane is usefulness for the microalgae culture in terms of the microalgal growth rate and the dissolution of the carbon dioxide.

\section{Acknowledgements}

The work has been partially supported by Okayama Foundation for Science and Technology.

\section{References}

1. Chisti Y (2007) Biodiesel from microalgae. Biotechnol Adv 25: 294-306.

2. Carvalho AP, Meireles LA, Malcata FX (2006) Microalgal reactors: a review of enclosed system designs and performances. Biotechnol Prog 22: 1490-1506.

3. Chaumont $D$ (1993) Biotechnology of algal biomass production: a review of systems for outdoor mass culture. J Appl Phycol 5: 593-604.

4. Ugwu CU, Aoyagi H, Uchiyama H (2008) Photobioreactors for mass cultivation of algae. Bioresour Technol 99: 4021-4028.

5. Hase R, Oikawa H, Sasao C, Morita M, Watanabe Y (2000) Photosynthetic production of microalgal biomass in a raceway system under greenhouse conditions in Sendai city. J Biosci Bioeng 89: 157-163.

6. Tredici MR, Materassi R (1992) From open ponds to vertical alveolar panels the Italian experience in the development of reactors for the mass cultivation of photoautotrophic microorganisms. J Appl Phycol 4: 221-231.

7. Hu Q, Guterman H, Richmond A (1996) A flat inclined modular photobioreactor for outdoor mass cultivation of photoautotrophs. Biotechnol Bioeng 51: 51-60.

8. Rubio FC, Ferna'ndez FGA, Sa'nchez Pe'rez JA, Garci 'a Camacho F Rubio FCM, et al. (1999) Prediction of dissolved oxygen and carbon dioxide concentration profiles in tubular photobioreactors for microalgal culture Biotechnol Bioeng 62: 71-86.

9. Harker M, Tsavalos AJ, Young AJ (1996) Autotrophic growth and carotenoid production of Haematococcus pluvialis in a 30 liter airliftphotobioreactor. J Ferment Bioeng 82: 113-118.

10. Ugwu CU, Ogbonna JC, Tanaka H (2002) Improvement of mass transfer characteristics and productivities of inclined tubular photobioreactors by installation of internal static mixers. Appl Microbiol Biotechnol 58: 600-607.

11. Kcmond A, Lichtenberg E, Stahl B, Vonshak A (1990) Quantitative assessment of the major limitations on the productivity of Spialina platensis in open raceways. J Appl Psychol 2: 195-206.

12. Oncel SS, Akpolat $O$ (2006) An integrated photobioreactor system for the production of Spirulina platensis. Biotechnology 5: 365-372

13. Kommareddy AR, Anderson GA (2003) Study of Light as a Parameter in the Growth of Algae in a Photo-Bio Reactor (PBR), ASAE Paper No. 034057 ASAE, St. Joseph, Michigan.

14. Bitog JP, Lee IB, Lee CG, Kim KS, Hwang HS, et al. (2011) Application of computational fluid dynamics for modeling and designing photobioreactors for microalge production: A reviw. J Comp Elect Agri 76:131-147.

15. Rahaman MSA, Cheng LH, Xu XH, Zhang L, Chen HL (2011) A review of carbon dioxide capture and utilization by membrane integrated microalgal cultivation processes. J Renew Sustain Ener Rev 15: 4002-4012

16. Chiu SY, Kao CY, Chen CH, Kuan TC, Ong SC, et al. (2008) Reduction of CO2 by a high-density culture of Chlorella sp. in a semicontinuous photobioreactor. Bioresour Technol 99: 3389-3396.

17. Hirata S, Hayashitani M, Taya M, Setsuji T (1996) Carbon dioxide fixation in batch culture of Chlorella sp. using a photobioreactor with a sunlight-collection device. J Ferment Bioeng 81: 470-2.

18. Carvalho AP, Malcata FX (2001) Transfer of carbon dioxide within cultures of microalgae: plain bubbling versus hollow-fiber modules. Biotechnol Prog 17: 265-272.

19. Gallagher SL, Tharakan JT, Chau PC (1987) An intercalatedspiral wound hollow fiber bioreactor for the culture of mammalian cells. Biotechnol Tech 1 : 91-96.

20. Chen F, Johns MR (1995) A strategy for high cell density culture of heterotrophic microalgae with inhibitory substrates. J Appl Phycol 7: 43-46.

21. Marsot P, Cembella AD, Mouhri K (1992) Croissance de la biomasse azote 
Citation: Sano Y, Horibe A, Haruki N, Okino Y (2016) Microalgal Culture for Chlorella sp. using a Hollow Fiber Membrane Module. J Membra Sci Technol 6: 147. doi:10.4172/2155-9589.1000147

Page 6 of 6

'e du Phaeodactylum tricornutum (Bacillariophyceae) en culture discontinue dialysante et non-dialysante. Can J Microbiol 38: 945-952.

22. Markov SA, Bazin MJ, Hall DO (1995) Hydrogen photoproduction and carbon dioxide uptake by immobilized Anabaena Variabilis in a hollow-fiber photobioreactor. Enzyme Microb Technol 17: 306-310.
23. Goldman JC, Dennett MR, Riley CB (1981) Inorganic Carbon Sources and Biomass Regulation in Intensive Microalgal Cultures. Biotechnol Bioeng 23 995-1014.

24. Becker EW (1994) Large-Scale Cultivation In Microalgae: Biotechnology and Microbiology, Becker E W, Cambridge University Press: UK pp: 63-171. 